\title{
Evaluation of Ti implants coated with Ag-containing borate bioactive glass for simultaneous eradication of infection and fracture fixation in a rabbit tibial model - ERRATUM
}

Wei Xiao, Shi-Hua Luo, Xia-Juan Wei, Chang-Qing Zhang, Wen-Hai Huang, Jia-Kang Chen, Yong Cai, Yong Rui, and Mohamed N. Rahaman

doi: 10.1557/jmr.2012.375, Published by Materials Research Society with Cambridge University Press, 5 December 2012.

In Xiao et al. ${ }^{1}$, it should be noted that the authors Wei Xiao and Shi-Hua Luo contributed equally to the work.

The publisher appreciates the opportunity for clarification.

\section{REFERENCE}

1. Wei Xiao, Shi-Hua Luo, Xia-Juan Wei, Chang-Qing Zhang, Wen-Hai Huang, Jia-Kang Chen, Yong Cai, Yong Rui, and Mohamed N. Rahaman: Evaluation of Ti implants coated with Ag-containing borate bioactive glass for simultaneous eradication of infection and fracture fixation in a rabbit tibial model. J. Mater. Res. 27(24), 3147-3156 (2012). 